\begin{tabular}{|c|c|}
\hline Title & Mott-Schottky Plot of the Passive Film Formed on Iron in Neutral Borate and Phosphate Solutions \\
\hline Author(s) & A zumi, Kazuhisa; Ohtsuka, Toshiaki; Sato, Norio \\
\hline Citation & $\begin{array}{l}\text { J. Electrochemical Society, 134(6), 1352-1357 } \\
\text { https://doi.org/10.1149/1.2100672 }\end{array}$ \\
\hline Issue Date & 1987-06 \\
\hline Doc URL & http://hdl.handle.net/2115/62192 \\
\hline Rights & $\begin{array}{l}\text { (c) The Electrochemical Society, Inc. 1987. A II rights reserved. Except as provided under U.S. copyright law, this work } \\
\text { may not be reproduced, resold, distributed, or modified without the express permission of The Electrochemical Society } \\
\text { (ECS). The archival version of this work was published in J. Electrochem. Soc. } 1987 \text { volume 134, issue 6, 1352-1357. }\end{array}$ \\
\hline Type & article \\
\hline File Information & J.Electrochem.Soc.134,1352-1357(1987), Mott-Schottky ---iron --.pdf \\
\hline
\end{tabular}

Instructions for use 


\title{
Mott-Schottky Plot of the Passive Film Formed on Iron in Neutral Borate and Phosphate Solutions
}

\author{
Kazuhisa Azumi, Toshiaki Ohtsuka, and Norio Sato* \\ Electrochemistry Laboratory, Faculty of Engineering, Hokkaido University, Sapporo, 060, Japan
}

\begin{abstract}
The semiconductive properties of the passive film formed on iron were investigated by measuring the impedance of passivated iron electrodes in neutral borate and phosphate solutions. Mott-Schottky type of plot was applied to the impedance data to obtain the donor density $N_{\mathrm{D}}$ and the flatband potential $V_{\mathrm{FB}}$ as a function of film formation potential, oxidation time, and ion species present in the solution. The value of $N_{1}$ obtained for the passive film is in the range of $1 \times 10^{26}-2 \times$ $10^{27} \mathrm{~m}^{-3}$ and decreases with increasing formation potential and oxidation time, indicating that the structural and electronic defects in the passive film decrease with increasing film thickness. The value of $N_{1}$ of the passive film formed in phosphate solution is greater than that in borate solution. From the fact that when the electrolyte was changed from borate to phosphate solution $N_{D}$ increased from the value in borate solution to the value in phosphate solution in a time period of about $10^{3} \mathrm{~s}$, it is evident that phosphate ions penetrate into the passive film producing a large number of defects.
\end{abstract}

Recent impedance (1-5) and photoelectrochemical (4, $5)$ investigations of the passive films on iron have suggested that the passivated iron behaves like a metal electrode covered with a n-type semiconductive oxide film. Stimming et al. $(1,2)$ conducted capacitance measurements of the passive film on iron and using the MottSchottky type of plot of the capacitance data claimed that the passive film was an $n$-type semiconductor with a high donor density of $10^{25}-10^{26} \mathrm{~m}^{-3}$. Khan and Schmickler (6) reported that the passive film was so thin that the simple Mott-Schottky plot might not be applicable, and proposed a model which took the surface charge of substrate metal into account to explain the capacitance data of an extremely thin semiconductor layer.

Recently, Stimming (7) has suggested that the amorphous nature of the passive film plays a significant role in the semiconductive properties of the films. Wheeler et al. (8) and Cahan et al. (9) have claimed, based on the capacitance-potential relation of the passive iron, that the chemisorbed substances, rather than the formation of the space charge layer in the film, have a great influence on the electric conductivity of the film.

In a previous study (10) the authors reported the impedance-potential relationship of the passive iron in a wide frequency region from $10^{-2}$ to $10^{4} \mathrm{~Hz}$ in borate and phosphate solutions at various $p H$ values. The results of the impedance response indicate that the passivated iron electrode has two time constants, one which appears in the frequency region lower than $10 \mathrm{~Hz}$ originates with the space charge in the film and the other appearing in the frequency region higher than $10 \mathrm{~Hz}$ may originate with the film/solution interface.

In this paper the Mott-Schottky type of plot is applied to the impedance data for studying the semiconductive property of the passive film on iron in borate and phosphate solutions at $p \mathrm{H} 6.5$ and $p H$ 8.4. The relationships between the donor density and the film formation potential as well as between the donor density and the kinds of anions present in electrolyte solution are discussed.

\section{Experimental}

A cylindrical electrochemical cell made of Pyrex glass was placed in a shield box. All electrical connections were made by a coaxial cable. A cylindrical platinized platinum plate form and a saturated calomel electrode (SCE) were used as a counterelectrode and a reference electrode, respectively. In this paper electrode potential is referred to the reversible hydrogen electrode ( $v s$. RHE).

The iron electrode used was prepared from a sheet of $99.99 \%$ Ferrovac-E iron. The electrode surface was mechanically polished by $0.05 \mu \mathrm{m}$ alumina abrasive and washed in ethyl alcohol by using an ultrasonic cleaner. The exposed area of the electrode to the electrolyte was

*Electrochemical Society Active Member. ca. $20 \mathrm{~mm}^{2}$ and the unexposed area was covered with silicon adhesive.

The solutions were mixtures of $0.30 \mathrm{~mol} \cdot \mathrm{dm}^{-3} \mathrm{H}_{3} \mathrm{PO}_{4}$ and $0.30 \mathrm{~mol} \cdot \mathrm{dm}^{-3} \mathrm{NaH}_{2} \mathrm{PO}_{4}(p \mathrm{H} 6.5)$, mixtures of 0.30 $\mathrm{mol} \cdot \mathrm{dm}^{-3} \mathrm{H}_{3} \mathrm{BO}_{4}$ and $0.075 \mathrm{~mol} \cdot \mathrm{dm}^{-3} \mathrm{Na}_{2} \mathrm{~B}_{4} \mathrm{O}_{7}(p \mathrm{H} 6.5$ and 8.4). All the solutions were prepared from doubly distilled water and analytical grade reagents, and deareated by bubbling purified nitrogen gas before experiments. All experiments were performed at a constant temperature of $298 \pm 0.5 \mathrm{~K}$ in the atmosphere of purified nitrogen.

A frequency response analyzer (FRA; NF Circuit Corporation, Model S-5720B) was used for the measurement of electrode impedance. The measurement was conducted by superimposing an ac voltage of $5 \mathrm{mV}$ amplitude with the frequency ranging from $1 \times 10^{-2}$ to $1 \times 10^{4}$ $\mathrm{Hz}$ on a dc bias of a potentiostat. A microcomputer (Nippon Electric Corporation, Model PC-9801) was used to control the FRA and the potentiostat, and to analyze the measured data.

In order to obtain the capacitance value of the spacecharge layer in the passive film a curve fitting method on the Bode diagram was employed (10). An example for the curve fitting is shown in Fig. 1, where an equivalent circuit is assumed consisting of a series connection of two parallel RC circuits and a resistance of solution $R_{\mathrm{S}}$. The $R_{\mathrm{SC}}$ and $C_{\mathrm{SC}}$ couple, which predominates in the frequency region lower than $10 \mathrm{~Hz}$, characterizes the property of the space charge layer in the passive film. The other couple, $R_{\mathrm{IF}}$ and $C_{\mathrm{IF}}$, which appears in the frequency region higher than $10 \mathrm{~Hz}$ corresponds to the film/solution interface. The validity of this equivalent circuit was discussed in the previous paper (10).

Passive films were formed by anodic oxidation (i) in $p H 8.4$ borate solution directly by polarizing cathodically reduced film-free surface and (ii) in $p H 6.5$ solutions in two-step oxidation (10) in order to prevent the surface from roughening during passivation. In the two-step oxidation, the iron electrode was first oxidized at $0.54 \mathrm{~V}$ in pH 8.4 borate solution for 600 s by potentiostatic oxidation to form a thin passive film and, after exchanging the solution to that of $p H 6.5$, it was further oxidized at a given potential.

To evaluate the semiconductive property of the passive film, the Mott-Schottky type of plot was made with the impedance data obtained by the procedure illustrated in Fig. 2. as described in the following:

1.The passive film was formed at a given potential, $V_{\mathrm{f}}$, for $1.8 \mathrm{ks}$.

2 . The potential was lowered to a potential, $V_{m(1)}$, where the impedance diagram was measured in the frequency range from $10^{-2}$ to $10^{4} \mathrm{~Hz}$ within $300 \mathrm{~s}$.

3 . The potential was then raised again to the potential, $V_{\mathrm{f}}$, and maintained there for $10 \mathrm{~s}$ and lowered to $V_{\mathrm{m}(2)}$ where the impedance diagram was measured. 


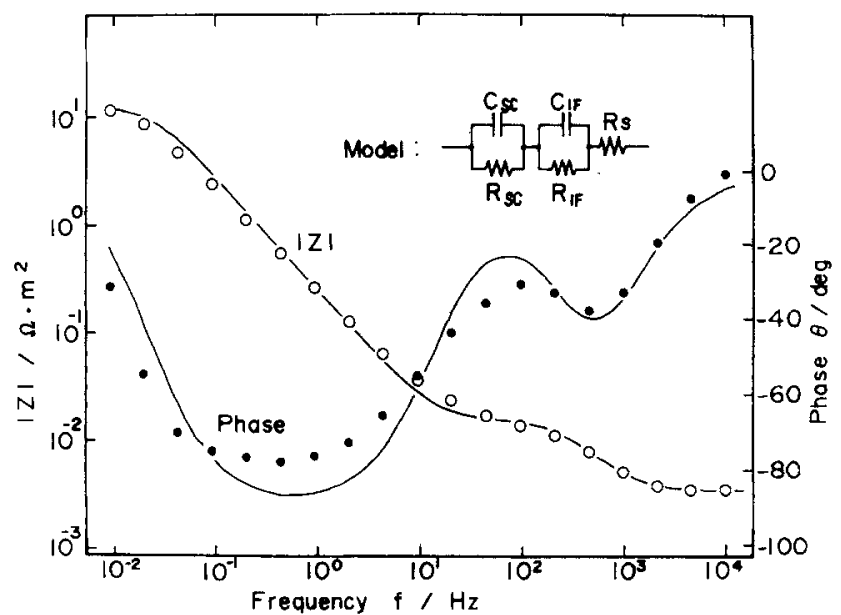

Fig. 1. Frequency dependence of impedance $(\log |Z|)$ and phase angle $(\theta)$ of iron electrode passivated at $0.63 \mathrm{~V}$ in $\mathrm{pH} 6.5$ borate solution. Solid line is a theoretical line calculated from the equivalent circuit shown in the figure.

4. Procedure 3 was repeated to obtain the impedance diagram at a successively lowering potential $V_{m(n)}$.

From the impedance diagrams obtained at various potentials $V_{\mathrm{m}}$ the components of $C_{\mathrm{Sc}}, R_{\mathrm{Sc}}, C_{\mathrm{FF}}, R_{\mathrm{IF}}$, and $R_{\mathrm{S}}$, can be estimated for the passive film formed at a certain potential $V_{\mathrm{r}}$. Finally, from $\mathrm{C}_{\mathrm{Sc}}{ }^{-2}$ changing with $V_{\mathrm{m}}$, one can obtain the Mott-Schottky plot for the passive film formed at various potentials $V_{f}$.

The thickness of the passive film formed on iron was measured by 3 parameter (3P) reflectometry (11-13).

\section{Results}

Relation between the capacitance of the space-charge layer and the formation potential.-The impedance diagram as shown in Fig. 2 was also measured for the passivated iron electrode after $1.8 \mathrm{ks}$ oxidation at various potentials in solutions. The value of capacitance $C_{s c}$ of the space-charge layer in the passive film formed in $p H$ 8.4 borate solution estimated from the diagram is shown in Fig. 3, where the reciprocal of the space-charge layer capacitance, $\mathrm{Csc}^{-1}$, is plotted as a function of potential. In Fig. 3 the electrode potential is raised in steps from 0.34 to $2.34 \mathrm{~V}$ to thicken the passive film. Impedance was measured at each potential step after holding the potential for $1.8 \mathrm{ks}$ before each measurement. It is seen that $C_{\mathrm{SC}}{ }^{-1}$ increases with potential in the passive potential region from 0.34 to $1.5 \mathrm{~V}$. At potentials $>1.5 \mathrm{~V}$, however, $\mathrm{C}_{\mathrm{SC}}{ }^{-1} \mathrm{de}-$ creases with potential.

Figure 4 shows the reciprocal of the space-charge layer capacitance measured at various potentials $V_{m}$ more negative than the film formation potential $V_{\mathrm{f}}$ for the passive

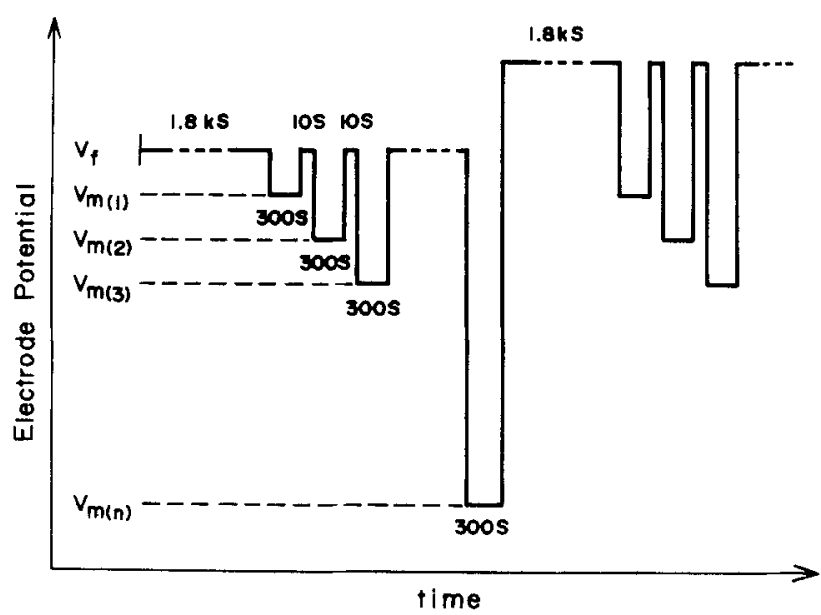

Fig. 2. Potential-time diagram for measurement of Mott-Schottky relation. $V_{\mathrm{f}}$ is film formation potential, $V_{\mathrm{m}}$ is measurement potential.



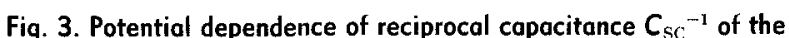
space-charge layer in the passive film formed on iron in $\mathrm{pH} 8.4$ borate solution $\left(V_{\mathrm{f}}=V_{\mathrm{m}}\right)$.

films formed at $V_{\mathrm{f}}=1.34$ and $2.34 \mathrm{~V}\left(V_{\mathrm{m}}<V_{\mathrm{f}}\right)$. For the passive film formed at $V_{\mathrm{f}}=1.34 \mathrm{~V} \mathrm{C}_{\mathrm{SC}}{ }^{-1}$ decreases with descending potential, while for the passive film formed at $V_{\mathrm{f}}=2.34 \mathrm{~V} \mathrm{C}_{\mathrm{Sc}^{-1}}$ first increases, reaches a maximum, and then decreases with descending potential $V_{\mathrm{m}}$. For the passive films formed at other potentials $V_{\mathrm{f}}$ the relationship obtained between $C_{\mathrm{sc}}{ }^{-1}$ and $V_{\mathrm{m}}$ was similar to that shown in Fig. 4. A peak of $\mathrm{C}_{\mathrm{SC}^{-1}}$ is always obtained in the $C_{S c^{-1}}{ }^{-1} V_{m}$ plot for the film formed at potentials more positive than $V_{\mathrm{f}}=1.5 \mathrm{~V}$, whereas a monotonous decrease of $\mathrm{C}_{s \mathrm{c}^{-1}}$ with lowering $V_{\mathrm{m}}$ is observed for the film formed at potentials more negative than $V_{\mathrm{f}}=1.5 \mathrm{~V}$.

The passive film on iron has been considered to be a semiconductor of n-type. The increasing anodic potential therefore induces the thickening of the space-charge layer as well as film thickening. In the case of $V_{m}=V_{f}$ (Fig. 3) the linear increase of $\mathrm{C}_{\mathrm{Sc}}{ }^{-1}$ with potential in the potential region less positive than $1.5 \mathrm{~V}$ implies the linear growth of the space charge layer accompanied with the film thickening. At potentials more positive than $1.5 \mathrm{~V}$, however, the $C_{\mathrm{sc}^{-1}}{ }^{-1}$ decreases with potential, although the film thickens further. To explain such a decrease of $C_{\mathrm{SC}}{ }^{-1}$ in the high potential region Stimming and Schultze (14) proposed the formation of an inversion layer in the passive film. Taking into account the fact that the peak

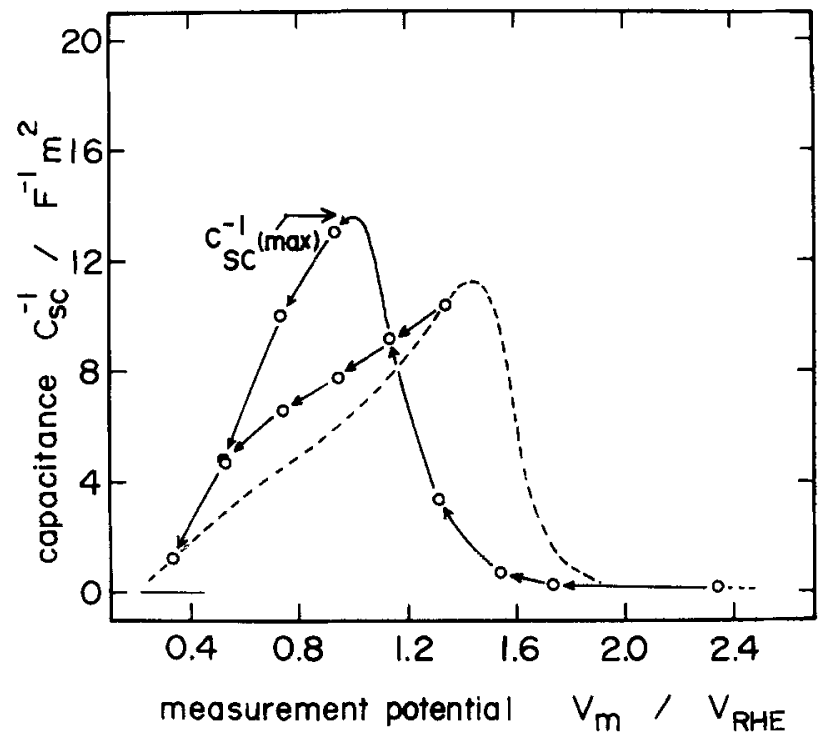

Fig. 4. Reciprocal capacitance $C_{\mathrm{SC}^{-1}}$ of the space-charge layer in the passive film in $\mathrm{pH} 8.4$ borate solution as a function of decreasing potentials, $V_{\mathrm{m}}\left(V_{\mathrm{m}}<V_{\mathrm{f}}\right)$. The passive film was formed at $V_{\mathrm{f}}=1.34 \mathrm{~V}$ and at $V_{\mathrm{f}}=2.34 \mathrm{~V}$ for $1.8 \mathrm{ks}$ 


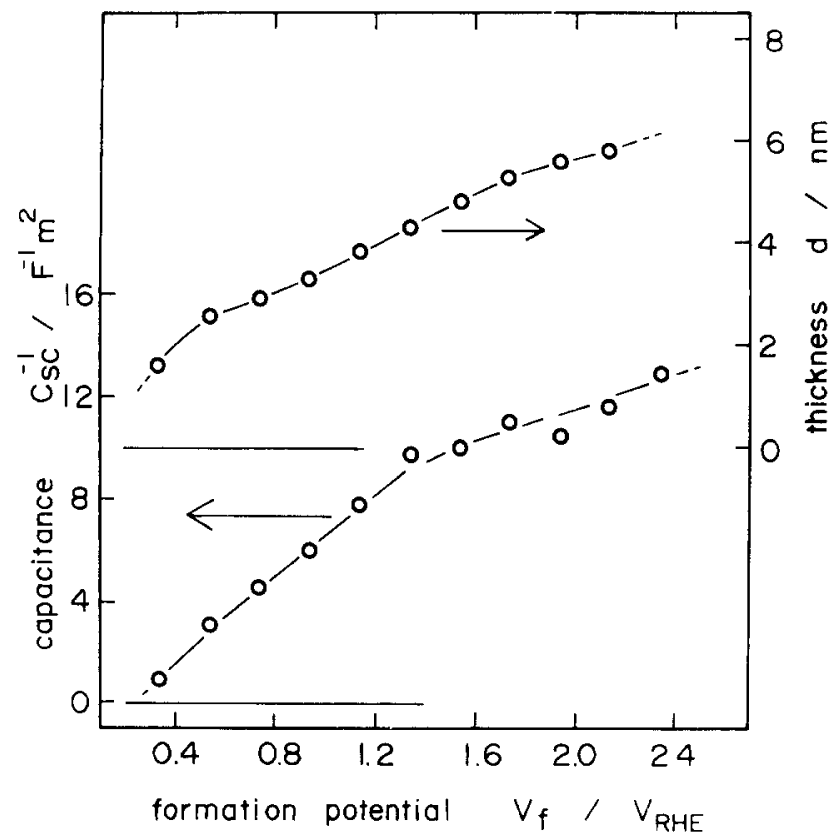

Fig. 5. Dependence of $\mathrm{C}_{\mathrm{Sc}}{ }^{-1}(\max )$ on the film formation potential measured for the passive film formed on iron in $\mathrm{pH} 8.4$ borate solution. Film thickness $d$ was measured by 3P-reflectometry $(11-13)$ under the same condition.

potential of $\mathrm{C}_{\mathrm{Sc}^{-1}}$ appears at $1.0 \mathrm{~V}$ in the $\mathrm{C}_{\mathrm{Sc}}{ }^{-1}-V_{\mathrm{m}}$ curve which differs from $1.5 \mathrm{~V}$ at which the peak of $\mathrm{C}_{\mathrm{SC}} \mathrm{C}^{-1}$ is observed in the $C_{\mathrm{SC}^{-1}}-V_{\mathrm{f}}$ curve, we propose that the surface states taking part in the anodic evolution of oxygen are responsible for the decrease of $\mathrm{C}_{\mathrm{Sc}^{-1}}{ }^{-1}$. The surface states may be produced by absorbed species such as $\mathrm{O}^{2-}(\mathrm{ad})$, $\mathrm{O}^{-}(\mathrm{ad})$, and $\mathrm{O}^{\prime \prime}(\mathrm{ad})$ which act as an intermediate of the oxygen evolution process. The adsorbed species or the surface states may be stable at potentials more positive than $1.0 \mathrm{~V}$, and therefore the peak of $\mathrm{C}_{\mathrm{sc}^{-1}}$ may appear around 1.0V as observed in Fig. 4.

The maximum value of $C_{\mathrm{sc}}^{-1}(\max )$ during the lowering of potential after the formation of the film at potentials more positive than $1.5 \mathrm{~V}$ can be regarded as corresponding to the maximum space charge layer thickness produced in the film. Figure 5 shows the maximum values of

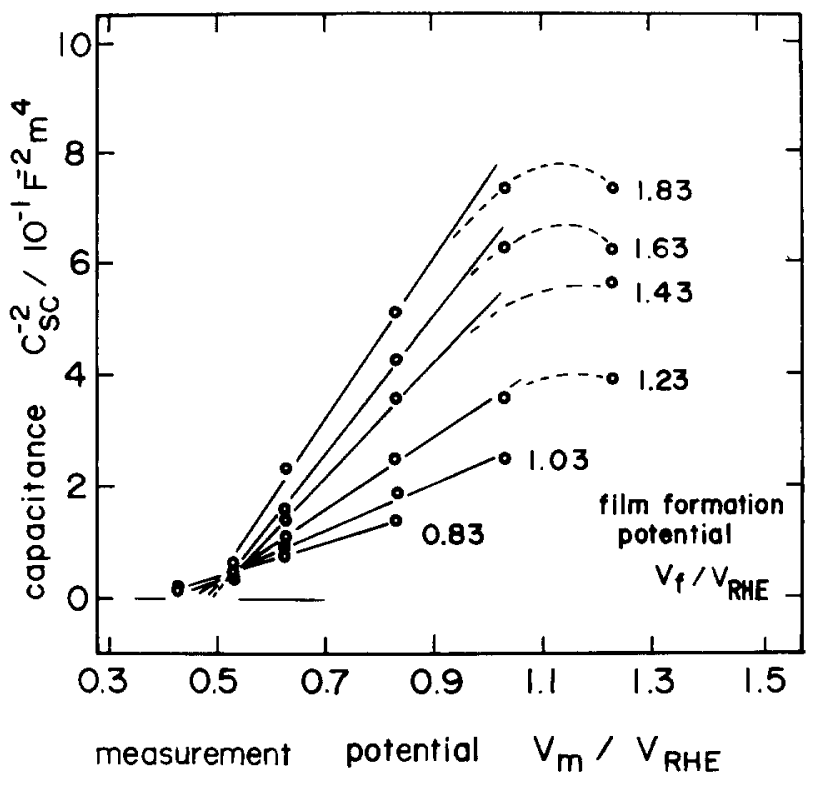

Fig. 6. The Mott-Schottky type of plot $\left(C_{\mathrm{SC}^{-}}{ }^{-2}\right.$ vs. $\left.V_{\mathrm{m}}\right)$ for the passive films formed at various potentials in $\mathrm{pH} 6.5$ borate solution for $1.8 \mathrm{ks}$.

$\left.C_{\mathrm{ST}^{-1}}{ }^{-1} \max \right)$ as a function of the formation potential, $V_{\mathrm{F}}$. In Fig. 5 the total thickness, $d$, of the passive film is also plotted for comparison. The thickness data are referred to in the previous study $(12,13)$ in which the thickness was estimated by reflectivity measurements. It is found from Fig. 5 that both $C_{\mathrm{Sc}^{-}}{ }^{-1}(\mathrm{max})$ and $d$ increase with ascending potential.

Mott-Schottky type of plot for the capacitance.-From the Mott-Schottky plot, which is shown in Fig. 6, the value of the donor density, $N_{\mathrm{D}}$, and the flatband potential, $V_{\mathrm{FB}}$, can be calculated by using the equation

$$
C_{\mathrm{SC}}{ }^{-2}=\left(2 / e N_{\mathrm{I}} \epsilon \epsilon_{\mathrm{H}}\right)\left(V_{\mathrm{m}}-V_{\mathrm{FB}}-k T\right)
$$

where $e$ is the charge of electron, $\epsilon_{0}$ the permittivity of the free space, $\epsilon$ the dielectric constant, $N_{1}$, the donor density, $V_{\mathrm{m}}$ the potentials where measurements are carried out, and $V_{\mathrm{FB}}$ the flatband potential. The donor density $N_{\mathrm{I}}$ is estimated from the slope and the flatband potential $V_{\mathrm{FB}}$ from extrapolation of $\mathrm{C}_{\mathrm{SC}^{-2}}{ }^{-2}$ to $\mathrm{C}_{\mathrm{SC}^{-2}}=0$. For
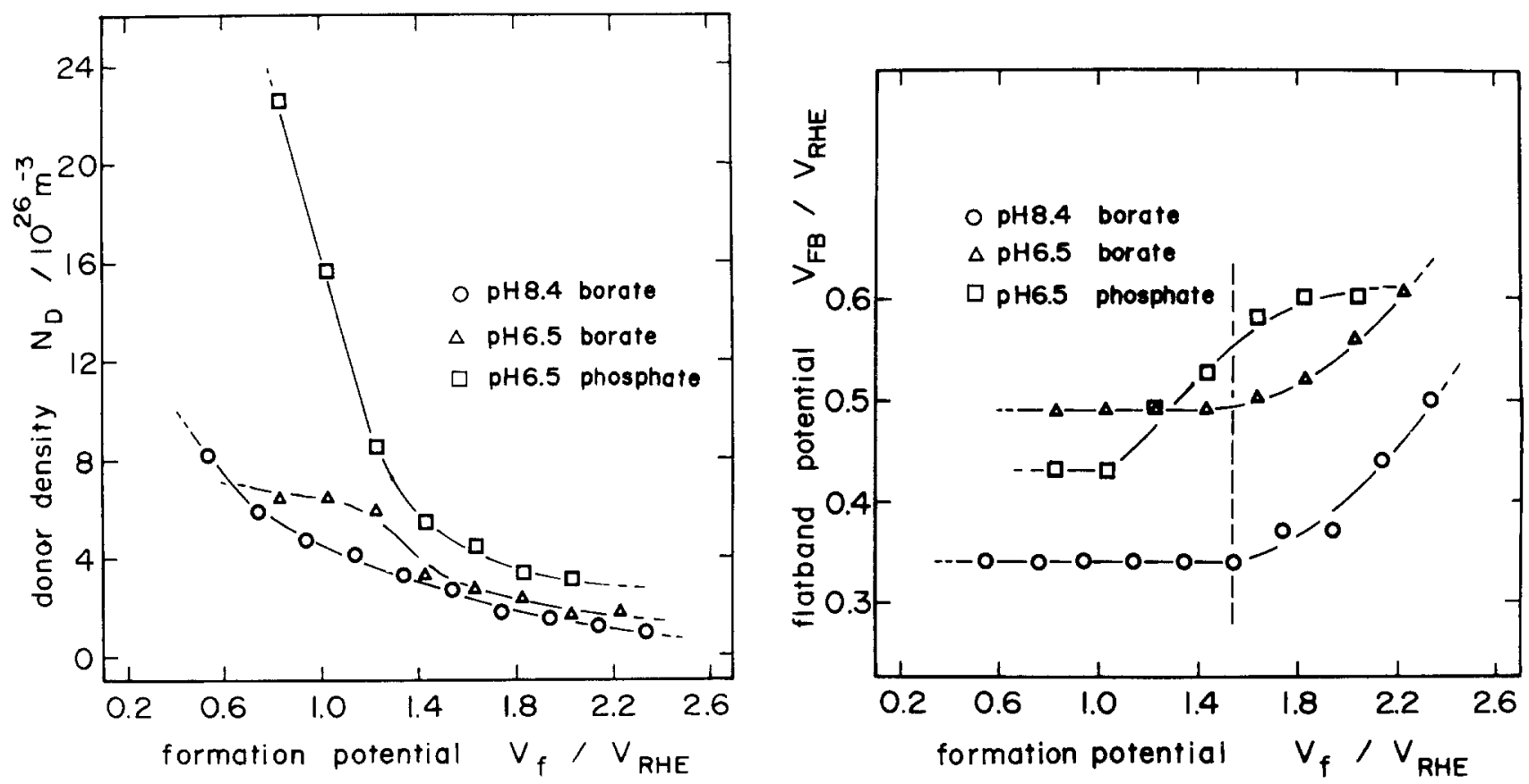

Fig. 7a. (a, left) Donor density $N_{D}$ of the passive film formed on iron in $p H ~ 8.4$ borate, 6.5 borate, and 6.5 phosphate solutions os a function of film formation potential. (b, right) Flatband potential $V_{\mathrm{FB}}$ of the passive film formed on iron in $p \mathrm{H} 8.4$ borate, $\mathrm{pH} 6.5$ borate, and pH 6.5 phosphate solutions as a function of film formation potential. 
calculation of $N_{\mathrm{D}}$, a value of dielectric constant $\epsilon=40$, which was previously estimated $(10)$, has been used for the passive film.

Figure 6 shows examples of the Mott-Schottky type of plot, which were obtained for the films formed at various potentials in $p \mathrm{H} 6.5$ borate solution. In Fig. 6 a linear relationship between $C_{\mathrm{Sc}}{ }^{-2}$ and $V_{\mathrm{m}}$ is found to hold in the potential region lower than $1.0 \mathrm{~V}$. Similar results to these shown in Fig. 6 were also obtained for the films formed in $p \mathrm{H} 8.4$ borate and $p \mathrm{H} 6.5$ phosphate solutions.

The donor density, $N_{\mathrm{D}}$, in the iron passive film formed in different solutions is shown in Fig. $7 \mathrm{a}$ as a function of the formation potential $V_{f}$. The value of $N_{\mathrm{D}}$ is estimated to be $1 \times 10^{26}-2 \times 10^{27} \mathrm{~m}^{-3}$, which is much greater than the value for ordinary semiconductors and iron oxides (3-5, 15-17). $N_{\mathrm{D}}$ decreases with ascending $V_{\mathrm{f}}$ in the three solutions. $N_{\mathrm{D}}$ also depends on the ion species present in the solution and the value of $N_{\mathrm{D}}$ in $\mathrm{pH} 6.5$ phosphate solution is greater than that in $p \mathrm{H} 6.5$ borate solution.

The flatband potential $V_{\mathrm{FB}}$ is found to depend to some extent on the film formation potential, as shown in Fig. $7 \mathrm{~b}$. In the potential region lower than $1.5 \mathrm{~V}$, the value of $V_{\mathrm{FB}}$ is constant at $V_{\mathrm{FB}}=0.34 \mathrm{~V}$ in $p \mathrm{H} 8.4$ borate solution and at $V_{\mathrm{FB}}=0.49 \mathrm{~V}$ in $p \mathrm{H} 6.5$ borate solution. In the potential region more positive than $V_{\mathrm{f}}=1.5 \mathrm{~V}$, the flatband potential shifts towards the more positive potential, as the film formation potential $V_{\mathrm{f}}$ becomes more positive. This apparent shift may be due to the influence of adsorbed oxygen species on the film resulting from the oxygen evolution reaction in the potential region more positive than $1.5 \mathrm{~V}$, as mentioned above. This shift of $V_{\mathrm{FB}}$ is seen to commence at a potential more negative than $1.5 \mathrm{~V}$ in phosphate solution, suggesting that besides adsorbed oxygen species phosphate ions adsorbed on or incorporated in the film play a certain role in shifting $V_{\mathrm{FB}}$.

Effect of the oxidation time.-The period of time for oxidation also influences the Mott-Schottky type of plot for the space-charge capacitance data. Figure 8 shows $N_{\text {D }}$ and $V_{\mathrm{FB}}$ as a function of oxidation time $t_{\mathrm{OX}}$ for the passive films formed at $V_{\mathrm{f}}=0.94$ and $1.34 \mathrm{~V}$ in $\mathrm{pH} 6.5$ borate solution by means of two-step oxidation, where a very thin film was first formed at $0.54 \mathrm{~V}$ for $600 \mathrm{~s}$ in $p \mathrm{H} 8.4$ borate so lution and then was thickened by further oxidation at the designated potential in $\mathrm{pH} 6.5$ borate solution. For rapid measurements of impedance, the potential was stepwise lowered in steps as soon as possible after a given oxidation time had passed at a constant potential and the impedance measurement was carried out at a fixed frequency of $f=1.00 \mathrm{~Hz}$. From the Mott-Schottky type of plot for the capacitance data thus measured at $1.00 \mathrm{~Hz}$ the donor density, $N_{\mathrm{D}}$, and the flatband potential, $V_{\mathrm{FB}}$, were determined as a function of oxidation time by assuming $\epsilon=40$. As shown in Fig. $8, N_{D}$ decreases with increasing $t_{\mathrm{Ox}}$, while $V_{\mathrm{FB}}$ remains constant during the prolonged oxidation.

\section{Discussion}

Dependence of $N_{\mathrm{p}}$ on the film formation potential.-As shown in Fig. 7a, the donor density $N_{\mathrm{D}}$ in the passive film formed on iron decreases with ascending film formation potential $V_{\mathrm{f}}$, and simultaneously the film thickness $d$ increases with ascending $V_{\mathrm{f}}$, as shown in Fig. 5. From this result $N_{\mathrm{D}}$ may be considered to depend on the film thickness, as will be discussed below.

The film thickness $d$ of the iron passive film is very thin and of the order of 1-5 nm in the passive potential region. The passive film is considered to consist of a few layers of the unit cells whose lattice constant is $0.8 \mathrm{~nm}$ for $\mathrm{Fe}_{2} \mathrm{O}_{3}$. In such a thin film many lattice defects or imperfections may be induced because of its thinness (18) These defects or imperfections would be reduced in number with increasing film thickness, as the passive film tends to take the more stable atomic structure, the thicker the film grows.

Film thickness d also increases with oxidation time $t_{0 \mathrm{x}}$ at constant potential in neutral and alkaline solutions (13). As shown in Fig. 8, the donor density decreases with increasing oxidation time at constant potential. This re

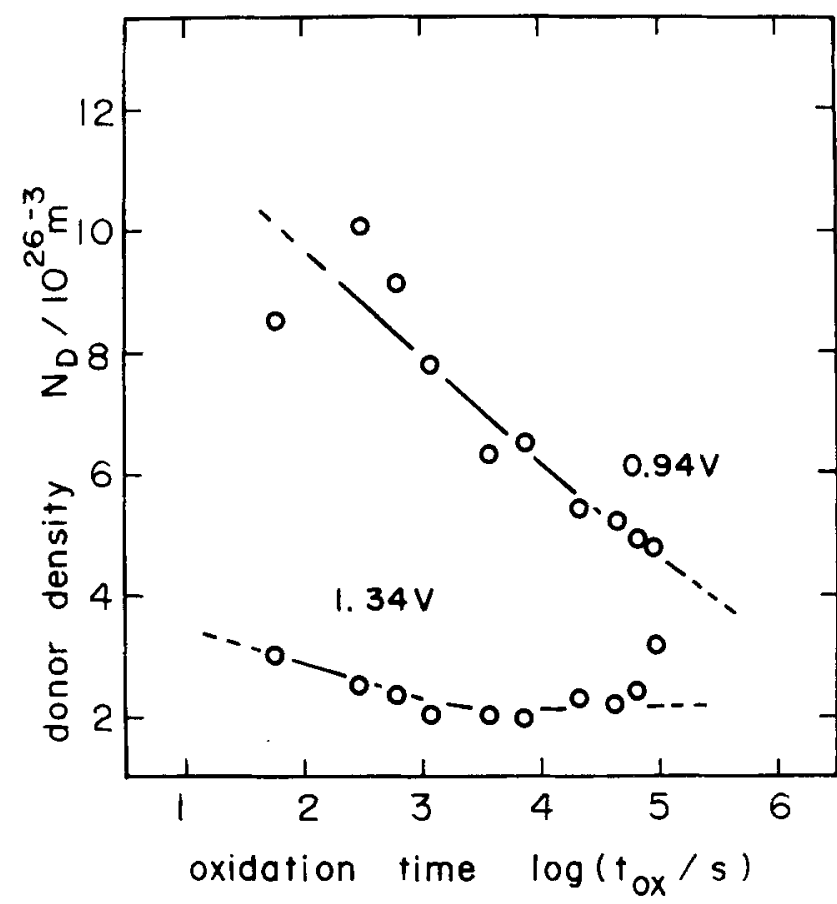

Fig. 8. Value of $N_{D} v s$. oxidation time $t_{0 X}$ for the passive film formed on iron at 0.94 and $1.34 \mathrm{~V}$ in $\mathrm{pH} 6.5$ borate solution. The impedance was measured at a fixed frequency of $1.00 \mathrm{~Hz}$.

sult also indicates that the density of the lattice defects acting as electron donors decreases with increase of the film thickness and with the aging of the film.

Effect of anion.-From comparison between the $N_{\mathrm{I}}$ value in phosphate solutions at $p \mathrm{H} 6.5$ and that in borate solutions at $p H 6.5$ (Fig. $7 a$ ), it is seen that the donor density is greater in the film formed in phosphate solution than in borate solution. Further, the change of $N_{\mathrm{D}}$ and $V_{\mathrm{FB}}$ during the second stage of the two-step oxidation in phosphate solution has been measured and compared with the result in Fig. 8. The result is given in Fig. 9, where the first oxidation was made at $E=0.54 \mathrm{~V}$ for $600 \mathrm{~s}$ in $p H 8.4$ borate solution, followed by the second stage of



Fig. 9. Chonge of $N_{D}$ and $V_{F B}$ with oxidation time $t_{0 X}$ for the passive film formed on iron at 0.94 and $1.34 \mathrm{~V}$ in $\mathrm{pH} 6.5$ phosphote solution. Impedance was measured at a fixed frequency of $1.00 \mathrm{~Hz}$. 
oxidation at $E=0.94$ or $1.34 \mathrm{~V}$ in $\mathrm{pH} 6.5$ phosphate solution. In Fig. 9 the change of $N_{\mathrm{D}}$ and $V_{\mathrm{FD}}$ is shown during the second stage of oxidation. In contrast with the result in Fig. 8, $N_{\mathrm{D}}$ in this case increases with oxidation time, in spite of the film increasing its thickness. The $V_{F B}$ also shifts from a value for the film in borate solution to one for the film in phosphate solution. The change of $N_{D}$ and $V_{F B}$ due to solution exchange requires a time period of about $10^{3} \mathrm{~s}$, and it is implied that the film slowly changes in its semiconductive property. The difference between the films formed in borate and the films in phosphate solution may be due to difference in the affinity of absorption in and/or adsorption on the film between these two anions. Phosphate ions probably possess the adsorption and absorption affinity greater than borate ions, resulting in the formation of iron phosphate compounds in the film $(19,20)$. From the results shown in Fig. 7, 8, and 9 the Mott-Schottky type of plot proves useful in studying the anionic effect on the passive film.

Thickness of the space charge layer.-The thickness $d_{\mathrm{SC}}$ of the space-charge layer in a semiconductor can be calculated by using the Schottky equation as follows

$$
d_{\mathrm{sc}}=\left(2 \epsilon \epsilon_{0} V_{\mathrm{Sc}} / e N_{\mathrm{D}}\right)^{1 / 2}
$$

where $V_{\mathrm{SC}}$ is the potential difference applied to the space-charge layer, which is assumed to be $V_{\mathrm{sc}}=V_{\mathrm{m}}-$ $\mathrm{V}_{\mathrm{FB}}$. According to Eq. [2] $d_{\mathrm{Sc}}$ can be estimated at each formation potential $V_{\mathrm{f}}$ from the data of $N_{\mathrm{D}}$ and $V_{\mathrm{FB}}$ shown in Fig. 7.

The thickness $d_{\mathrm{SC}}$ calculated from Eq. [2] as a function of $V_{\mathrm{f}}$ for the passive film formed in $\mathrm{pH} 6.5$ borate solution is shown in Fig. 10. In the figure the thickness $d^{\prime}{ }_{\mathrm{sc}}$ of the dielectric layer in the film is also shown for comparison, which was estimated from the capacitance data of $\mathrm{C}_{\mathrm{SC}^{-1}}(\max )$ shown in Fig. 5 with a parallel capacitor model $(14,21)$ (Eq. [3])

$$
d^{\prime} s^{\prime}=\epsilon \epsilon_{0} / C_{S(}(\max )
$$

where $d^{\prime}{ }_{S c}$ is regarded as the thickness of the dielectric layer in the passive film. In Fig. 10 the thickness data obtained from the reflectivity measurements (13) are also plotted.

In Fig. 10 both $d_{\mathrm{sc}}$ and $d_{\mathrm{Sc}}^{\prime}$ increase with increasing $d$ in the passive potential region. From comparison between the total thickness, $d$, and the thickness of the space charge layer, $d_{s c}$, it is suggested that the film con-

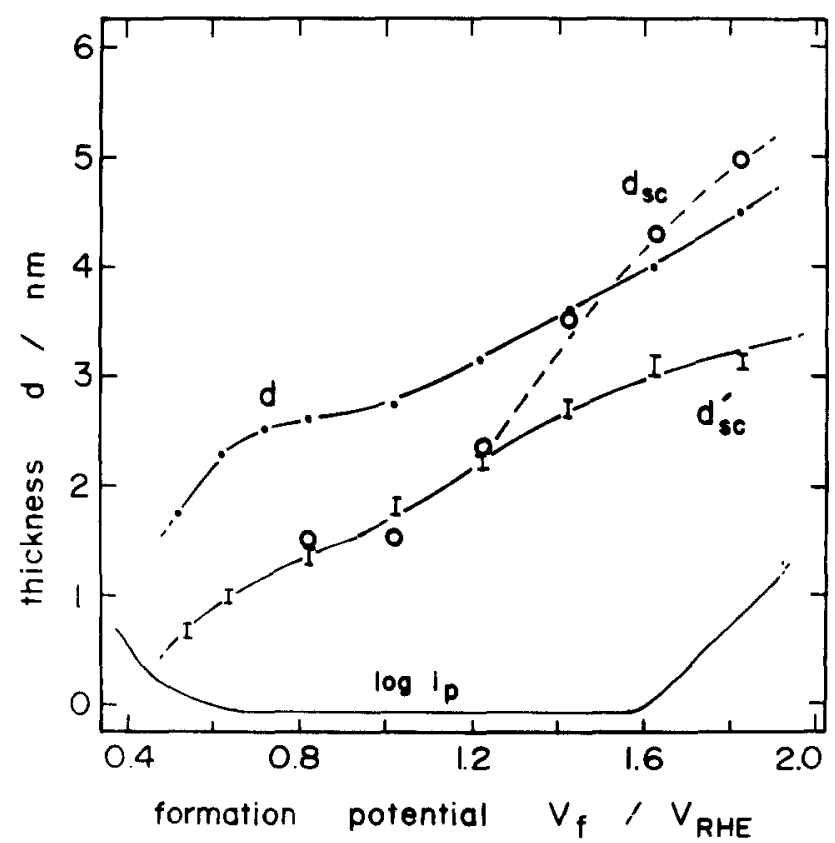

Fig. 10. Thickness $d_{\mathrm{SC}}$ of the space-charge layer calculated from Eq. [2] and [3] as a function of film formation potential. The passive film was formed in $p \mathrm{H} 6.5$ borate solution. The thickness of the passive film, $d$, measured by 3P-reflectometry $(12,13)$ was plotted for comparison. sists of two layers in the passive potential region. In the inner layer no electric field exists, while the outer layer contains the space charge and sustains a potential drop in itself. As seen in Fig. 10 the thickness of the inner layer is nearly constant in the passive potential region, independent of the formation potential. The constant thickness of the inner layer agrees with the assumption mentioned in the previous work (10). In the potential region of the oxygen evolution $d_{\mathrm{Sc}}$ is thicker than the real thickness. The thickness $d_{\mathrm{SC}}$ in the potential region, however, does not represent the real feature of the spacecharge, because the poiential may not be pinned by the Fermi level but by the surface states produced by the adsorbed oxygen species.

Khan and Schmickler (6) have suggested in explaining the capacitance data of a thin semiconductive film such as the iron passive film that the electrode capacitance is influenced not only by the space charge layer in the semiconductor but also by the surface charge on metal. A calculation based on the model of Khan and Schmickler was performed to obtain the solution of $N_{D}$ for the capacitance data in the present work. The result of this calculation, however, shows that the surface charge on metal may play no significant role in the capacitance of the passivated iron with roughness factor 1 .

\section{Conclusion}

The n-type semiconductive property of the passive film on iron was evaluated from the Mott-Schottky type of plot for the impedance data obtained. The following conclusions may be drawn from the results.

1 . The value of donor density $N_{\mathrm{D}}$ of the passive film is in the range of $1 \times 10^{26}-2 \times 10^{27} \mathrm{~m}^{-3}$ and decreases with film formation potential. This result suggests that the structural and electronic defects which provide donor centers in the passive film decrease with increasing film thickness.

2. The value of $N_{\mathrm{D}}$ and $V_{\mathrm{FB}}$ are affected by ionic species present in the solution. The passive film formed in phosphate solution possesses $N_{D}$ which is greater than that in borate solution. Phosphate ions may penetrate into the passive film, producing a large number of structural and electronic defects in the film compared with borate ion.

3. The flatband potential $V_{\mathrm{FB}}$ is estimated at $0.34 \mathrm{~V}$ in $\mathrm{pH}$ 8.4 borate solution and at $0.49 \mathrm{~V}$ in $p \mathrm{H} 6.5$ borate solution in the passive potential region and shifts to more positive potential in the potential region where oxygen evolution occurs. In $p H 6.5$ phosphate solution the flatband potential shifts to more positive potential through the passive and oxygen evolution potential regions.

Manuscripts submitted Sept. 8, 1986; revised manuscript received Dec. 30, 1986.

Hokkaido University assisted in meeting the publication costs of this article.

\section{REFERENCES}

1. U. Stimming and J. W. Schultze, Electrochim Acta., 24, 859 (1979).

2. U. Stimming, in "Passivity of Metals and Semiconductors," M. Froment, Editor, p. 477, Elsevier Pub. Co., Amsterdam (1983).

3. F. M. Delnick and N. Hackerman, This Journal, 126, $732(1979)$

4. S. M. Wilhelm, K. S. Yun, L. W. Ballenger, and N. Hackerman, ibid., 126, 419 (1979).

5. S. M. Wilhelm and N. Hackerman, ibid, 128, 1668 (1981).

6. S. U. M. Khan and W. Schmickler, J. Electroanal. Chem. Interfacial Electrochem., 108, 329 (1980).

7. U. Stimming, Electrochim Acta, 31, 415 (1986).

8. D. J. Wheeler, B. D. Cahan, C. T. Chen and E. Yeager, in "Passivity of Metals," R. F. Frankenthal and J. Kruger, Editors, p. 546, The Electrochemical Society Softbound Proceedings Series, Princeton, NJ (1978).

9. B. D. Cahan and C. T. Chen, This Journal, 129, 474 (1982).

10. K. Azumi, T. Ohtsuka, and N. Sato, Trans. Jpn. Inst. Met., 27, 382 (1986).

11. T. Ohtsuka and K. E. Heusler, J. Electroanal. Chem. Interfacial Electrochem., 100, 319 (1979); K. E. Heusler and T. Ohtsuka, Surf. Sci., 101, 194 (1980). 
12. K. Azumi, T. Ohtsuka, and N. Sato, Denki Kagaku, 53, $306(1985)$

13. K. Azumi, T. Ohtsuka, and N. Sato, ibid., 53, 700 (1985).

14. U. Stimming and J. W. Schultze, Ber. Bunsenges. Phys. Chem. 80, 1297 (1976)

15. R. A. Fredlein and A. J. Bard, This Journal, 126, 1892 (1979).

16. J. H. Kennedy and K. W. Frese, Jr., ibid., 125, 723 (1978).

17. G. Horowitz, J. Electroanal. Chem. Interfacial
Electrochem., 159, 421 (1983).

18. C. Y. Chao, L. F. Lin, and D. D. Macdonald, This Journal, 128, 1187 (1981); L. F. Lin, C. Y. Chao, and D. D. Macdonald, ibid., 128, 1194 (1981).

19. R. Nishimura and N. Sato, Bousyoku Gijutu, 26, 305 (1977).

20. K. Tokugawa, Jpn. J. Appl. Phys., 21, 1693 (1982); and ibid., 21, 1700 (1982).

21. R. V. Moshtev, Ber. Bunsenges. Phys. Chem., 72, 452 (1968).

\title{
A Mathematical Model for the Corrosion of Iron in Sulfuric Acid
}

\author{
E. C. Gan* and Mark E. Orazem ${ }^{\star *}$
}

Department of Chemical Engineering, University of Virginia, Charlottesville, Virginia 22903

\section{ABSTRACT}

A mathematical model is developed for the corrosion of a rotating iron disk in sulfuric acid. The model treats explicitly the coupling of interfacial reactions with the mass transfer of ionic species by migration, diffusion, and convection in both the diffuse part of the double layer and the diffusion layer. The corrosion reactions take place at the metal-electrolyte interface and are characterized by the interactions among heterogeneous reactions. The total current density at an electrode is obtained by summing the partial current densities due to each of these individual heterogeneous reactions. The homogeneous partial dissociation of sulfuric acid is also treated explicitly. This model shows that the mass-transferlimited currents can be attributed to mass-transfer limitations to the removal of corrosion products from the iron surface coupled with a reduction of the active area of the iron disk. The limiting current obtained from this model is proportional to the square root of the rotation speed and agrees with published experimental results.

The fundamental reaction for iron corrosion involves dissolution of the metal atoms into their ions. Although their reaction alone does not reflect the complexity of the iron corrosion process, mathematical models of this process are generally based upon this simplitied view. Griffin (1), for example, assumed competitive adsorption between an isolated cation and a cation in the oxide layer to model the active-passive transition. These cations were assumed to be the product of the electrode dissolution. With this simple kinetic model, he was able to reproduce qualitatively the "multiplicity of steady states" in the region prior to passivation. In the model by Law and Newman (2) a modified Butler-Volmer relationship was applied to express the simple iron dissolution reaction. Despite the simplicity assumed for the corrosion chemistry, the model provided good account for the $\mathrm{ki}$ netic resistance in the double layer and the nonuniform potential distribution across the disk surface. The concentration dependence of a limiting reactant was included in their kinetic expression in order to treat the effect of mass-transfer limitation. Epelboin et al. (3) suggested that the mass-transfer-limiting species might be the $\mathrm{OH}^{-}$species. This is unlikely, however, in an acidic medium which lacks the hydroxide ion concentration required to justify a significant involvement of this species in the passivation process. Alkire and Cangellari (4) reported the importance of certain chemical species by arguing that the impairment of its concentration buildup due to the influence of fluid flow impeded passivation. They indicated a critical velocity above which passivation did not occur. Russell and Newman (5) described a model for the iron corrosion in sulfuric acid which also included the formation and growth of a porous salt film. By using a simple electrode dissolution reaction and expressing it in the Butler-Volmer form as used by Law and Newman (2), the model provided a qualitative account of the processes leading to the formation of the salt film.

The principle advantage of the relatively simple mathematical descriptions given above is that unknown parameters are lumped to provide a minimal number of $\mathrm{ki}$ netic parameters. A more complete characterization of corrosion mechanisms requires treatment of multiple re-

\footnotetext{
* Electrochemical Society Student Member
}

** Electrochemical Society Active Member. actions. A general treatment of multiple electrode reactions by White et al. (6) allowed prediction of the total current density at an electrode under potentiostatic control. Treatment of multiple reactions was also expressed in the mathematical modeling of LiAl/FeS battery by Pollard and Newman (7).

In this work, the complex reactions at the electrode surface are treated by the coupling among simple reaction steps and mass transfer to and from the electrode surface. This approach incorporates both the macroscopic transport phenomena in the electrolytic solution and the microscopic model of the metal-electrolyte interface, allowing explicit treatment of the chemical species involved in the system. Passivation is considered in this work to be the formation of a protective oxide layer which reduces the active fraction of the surface. Progressive coverage by oxides has been observed by Miller (8) on an iron disk below the passivation potential. Through this approach, the influence of mass transfer on the corrosion current can be characterized without assumption of a mass-transfer-limited reactant in solution.

\section{Physical Description}

A one-dimensional schematic representation of the metal-electrolyte system is presented in Fig. 1. The electrolytic solution was divided into a diffusion layer that adjoins the bulk phase and diffuse part of the double layer, a relatively small region extending from the imaginary outer Helmholtz plane. Unlike the diffusion layer, the diffuse part of the double layer is not electrically neutral. The mathematical depiction of the metal-electrolyte interface was based on the theory of diffuse-double-layer as developed by Stern, Gouy, and Chapman [see, for example, Parsons (9)]. The microscopic model of the metalelectrolyte interface (shown in Fig. 1) included three planes: the inner and outer Helmholtz planes on the electrolytic solution adjacent to the interface and the inner surface state on the metal side. The inner surface state (ISS) was designated to be the plane associated with reactive metal atoms. The inner Helmholtz plane (IHP) is the locus of the centers of the first row of ions specifically adsorbed onto the metal surface. The outer Helmholtz plane (OHP) is the plane of closest approach for the solvated ions associated with the bulk solution. The charge held within the diffuse part of the double layer is balanced by the charge held at the interfacial planes IHP 